\title{
Relocalization switch in a triple quantum dot molecule in $2 \mathrm{D}$
}

\author{
Miloslav Znojil \\ The Czech Academy of Sciences, Nuclear Physics Institute, Hlavní 130, 25068 Rež, Czech \\ Republic \\ and \\ Department of Physics, Faculty of Science, University of Hradec Králové, \\ Rokitanského 62, 50003 Hradec Králové, Czech Republic \\ e-mail: znojil@ujf.cas.cz
}

\section{Keywords:}

coupled quantum dots in 2D;

probability density localization;

non-separable polynomial model;

quantum catastrophe control;

\section{PACS number:}

PACS 03.65.Ge - Solutions of wave equations: bound states

\begin{abstract}
A symmetric chain of three quantum dots (i.e., one of the simplest quantum dot molecules) is constructed using a three-parametric non-separable version of an asymptotically separable sextic polynomial potential $V(x, y)=\left(x^{2}+y^{2}\right)^{3}+\ldots$. The probability density $|\psi(x, y)|$ (admitting either the central or off-central dominance) is assumed measured. A dynamical regime is found with an enhanced sensitivity of the central - off-central transition to the parameters. Quantitatively, the possibility of control of such a switch alias "relocalization catastrophe" is illustrated nonnumerically.
\end{abstract}




\section{Introduction}

In the unitary version of quantum mechanics of confined systems one encounters a wide methodical gap between their one-dimensional subfamily described by Schrödinger equation

$$
\left[-\frac{\hbar^{2}}{2 \mu} \frac{d^{2}}{d x^{2}}+V(x)\right] \psi_{n}(x)=E_{n} \psi_{n}(x), \quad n=0,1, \ldots
$$

and various more general scenarios. They may be sampled by the very next 2D family described by partial differential Schrödinger equation

$$
\left[-\frac{\hbar^{2}}{2 \mu_{x}} \frac{d^{2}}{d x^{2}}-\frac{\hbar^{2}}{2 \mu_{y}} \frac{d^{2}}{d y^{2}}+V(x, y)\right] \psi_{m}(x, y)=E_{m} \psi_{m}(x, y), \quad m=0,1, \ldots
$$

The gap involves not only constructive mathematics (which is perceivably more complicated in the latter case) but also experimental physics where, on the contrary, the realization of the confinement is usually more challenging in the one-dimensional setup.

We believe that the transition from the most popular 1D Eq. (1) to the non-separable twodimensional bound-state problem (2) may be more straightforward than expected. In an analysis of this possibility we feel motivated by the results of our recent paper [1]. Indeed, for multiple polynomial multi-well one-dimensional potentials $V(x)$ we managed to localize there the existence of experimentally interesting bifurcations of probability densities $\left|\psi_{n}(x)\right|$. Moreover, we revealed that the theoretical prediction of such a "quantum catastrophic" phenomenon may remain nonnumerical or, at worst, semi-numerical.

In our present letter we intend to show that a similar successful determination of the sets of the critical coupling constants may be also achieved and performed in 2D.

\section{Classical versus quantum catastrophes}

Stable stationary equilibria of a classical dynamical system $\mathcal{S}$ may be identified with the minima of an ad hoc potential $V=V_{\mathcal{S}}(x)$ or $V=V_{\mathcal{S}}(x, y)$, etc. The dependence of properties of these equilibria on parameters was given an elementary qualitative classification by $\mathrm{R}$. Thom [2]. In applications [3] he proposed to speak about the "fold catastrophe" (using the benchmark choice of $V^{(f o l d)}(x)=x^{3}+a x$ characterized by a bifurcation of equilibria at $a=0$ ), about the "cusp catastrophe" (using $V^{(\text {cusp })}(x)=x^{4}+a x^{2}+b x$ ), etc. Naturally, the transparent geometric nature as well as the conceptual simplicity made this classification scheme useful in diverse areas ranging from the evolution scenarios in biology [4] up to various phenomenological aspects of quantum chemistry [5]. 
In [1] we pointed out that after a tentative transition to quantum systems $\mathcal{Q}$ and to the related bound-state Schrödinger Eqs. (11) or (2), the spectrum need not remain discrete (and/or bounded below) so that the paradigm (i.e., the Thom's classification scheme) must be amended. We emphasized that after quantization one has to omit, first of all, all of the "non-confining" Thom's potentials, i.e., not only the above-mentioned odd-parity function $V^{(f o l d)}(x)$ but also another one-dimensional potential $V^{\text {(swallowtail })}(x)=x^{5}+a x^{3}+b x^{2}+c x$ as well as all of the three two-dimensional "umbilic" benchmarks $V(x, y)$.

As a result, the Thom's menu of seven basic dynamical scenarios of an immediate classical applicability degenerates to the mere doublet of one-dimensional quantum potentials in Eq. (1) formed by the above-mentioned two-parametric function $V^{(\text {cusp })}(x)$ and/or by the four-parametric function

$$
V^{(\text {butterfly })}(x)=x^{6}+a x^{4}+b x^{3}+c x^{2}+d x .
$$

Moreover, in the context of quantum physics one also has to consider the effect of tunneling. At a negative $a<0$ in $V^{(\text {cusp })}(x)$, for example, the particle may be detected localized near both of the minima separated by the barrier. One can conclude that only the choice of $V^{(b u t t e r f l y)}(x)$ makes good sense and can be made responsible for simulation of a nontrivial observable quantum catastrophe.

In [1] we analyzed the butterfly model (3) and emphasized the existence of a change-oflocalization catastrophe during which the dominance of the central local minimum at $x=0$ becomes replaced by the topologically non-equivalent dominance of the two "remote" minima. Under a simplifying assumption of vanishing $b=d=0$ we were able to interpret such a "quantum catastrophe" as a phenomenon of spatial bifurcation of the probability density $|\psi(x)|$.

In applications, unfortunately, the existence of the formal theoretical and mathematical merits of the one-dimensional model may be found accompanied by the emergence of a certain discomfort in experimental setting. Indeed, the schematic one-dimensional, $y$-independent interactions $V(x, y)=V^{(\text {butterfly) }}(x)$ (admitting the free motion along the $y$-axis) have to be realized, in the laboratory, typically, by the parallel waveguides which have to be very long (i.e., in principle "infinitely" long).

Naturally, one of the most natural ways of suppression of such an obvious phenomenological disadvantage can and should be sought via a transition to the genuine two-dimensional models. In such a direction of research we were mainly encouraged by the observation that after an extension of our study [1] to 2D, the underlying Schrödinger Eq. (2) happened to be, in the dynamical regimes of interest, by far not that difficult to solve and analyze, in spite of its non-separability.

In our present letter a certain user-friendly family of models based on 2D potentials $V(x, y)$ will be proposed and considered, therefore. Briefly, these models may be characterized by the survival 
of the two basic mathematical merits of their 1D predecessors. The first one is mathematical, lying in the elementary non-numerical feasibility of an exact localization of the pronounced couplingdependent minima and maxima of the potentials in both of their 1D and 2D exemplifications. The second key merit of the present $2 \mathrm{D}$ extension of the family of tractable models is more pragmatic. Its essence (to be explained below) lies in the purely empirical observation that the above-mentioned determination of the "quantum catastrophic" dynamical regime becomes facilitated by the unexpectedly user-friendly nature of the bifurcation phenomenon which appears "abrupt", restricting the breakdown of the non-numerical tractability of the transition process to a very narrow interval of the couplings in both of the $1 \mathrm{D}$ and $2 \mathrm{D}$ models.

\section{Quantum bound states in 2D}

\subsection{Asymptotically separable toy-model potential and its extremes}

Although the Thom's classical theory does not offer, in two dimensions, any obvious methodical guidance, we intend to use a modified idea of Ref. [1]. By itself, it was inspired by the Arnold's extension [6] of the Thom's list of the specific one-dimensional benchmarks $V(x)$ to the higherdegree polynomials. This means that in what follows we shall also admit an arbitrary polynomial form of $V(x, y)$. Still, for the sake of brevity we shall only restrict our attention to the sufficiently elementary three-parametric two-dimensional model of the strictly confining polynomial form

$$
V(x, y)=r^{6}-A^{2} r^{4}+\left(B^{2}+C^{2} y^{2}\right) r^{2}, \quad r^{2}=x^{2}+y^{2} .
$$

At $y=0$ this function formally degenerates to the first nontrivial two-barrier model of Ref. [1] so that we may reparametrize its first two coupling constants,

$$
A^{2}=3\left(\alpha^{2}+\beta^{2}\right), \quad B^{2}=3 \alpha^{2} \gamma^{2}, \quad \gamma^{2}=\alpha^{2}+2 \beta^{2}
$$

According to section \# 3 in [1] the three local minima of function (4) do exist and do lie at $r=0$ (with vanishing value of $V(0,0)=0$ ) and at $(x, y)=( \pm \gamma, 0)$ (where we have $V( \pm \gamma, 0)=$ $\left.\left(\alpha^{2}-\beta^{2}\right) \gamma^{4}\right)$. The height of the local saddle-point-shaped barriers is given by formula $V( \pm \alpha, 0)=$ $\alpha^{4}\left(\beta^{2}+\gamma^{2}\right)$.

For the large values of parameters $\alpha$ and $\beta$ (with even larger $\gamma=\gamma(\alpha, \beta)$ ) the barrier is high so that the three local minima (all occurring at $y=0$ ) are well separated. In such a dynamical regime our present 2D quantum model may be perceived as qualitatively fully analogous to its 1D predecessors of Ref. [1. Not quite expectedly, also the leading-order determination of the low-lying spectra of quantum bound-state energies will be shown to remain fully analogous and manifestly non-numerical. 


\subsection{The mechanism of relocalization of $2 \mathrm{D}$ probability densities}

The ground state wave function may be found localized either near $x=r=0$ (provided that we have $\alpha \gg \beta$ ), or near the two separate centers with $x= \pm \gamma$ (provided that $\alpha \ll \beta$ ). A sudden "relocalization" alias catastrophic transition between these two topologically non-equivalent distributions of probability of finding the particle should finally be observed at $\alpha \approx \beta \gg 1$.

\subsubsection{Dynamical regime of centrally localized probability densities}

We have to remind the readers here that as long as we speak about the qualitative features of a non-trivial, 2D quantum system, the determination of the probability densities $|\psi(x, y)|$ obtainable from the manifestly non-separable Schödinger Eq. (21) is a truly challenging task requiring an application of the standard numerical construction techniques in general. Fortunately, once we restrict attention to the domain of large parameters $\alpha$ and $\beta$, our qualitative understanding of the dynamics becomes thoroughly simplified, based on the generic graphical illustration of the shape of $V(x, y)$ as provided by Fig. 1 .

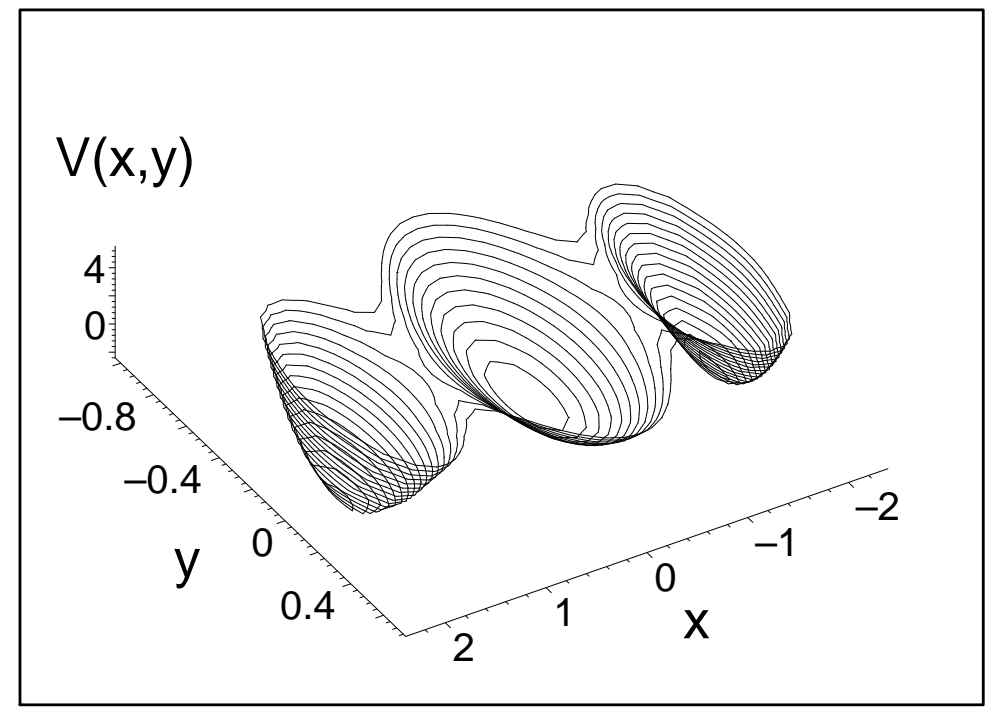

Figure 1: Sample potential $V(x, y)=\left(x^{2}+y^{2}\right)^{3}-6.6\left(x^{2}+y^{2}\right)^{2}+\left(10.2+9 y^{2}\right)\left(x^{2}+y^{2}\right)$ near its three local minima. Parameters are $\alpha^{2}=1, \beta^{2}=1.2$ and $\gamma^{2}=3.4$, the two outer minima are $V( \pm \gamma, 0)=-2.312$, while the heights of the two saddle-point barriers are $V( \pm \alpha, 0)=3.4$.

In the picture we see that in the vicinity of the origin the shape of the potential acquires the elementary analytic representation given directly by Eq. (4),

$$
V(x, y)=3 \alpha^{2} \gamma^{2} r^{2}+\mathcal{O}\left(r^{4}\right)
$$


Whenever we have, by our overall assumptions, $\alpha \gg \beta \gg 1$, the value of potential $V(0,0)=0$ will represent a pronounced global minimum of its shape.

The most important feature of the shape of $V(x, y)$ near this minimum is its central symmetry. The potential becomes very well approximated by the exactly solvable harmonic-oscillator well. Thus, one immediately obtains the leading-order formula for energies

$$
E_{n}^{(0)}=2 \sqrt{3}(2 n+1) \alpha \gamma+\ldots \quad n=0,1, \ldots .
$$

This formula may be then used as an approximation of the low-lying part of the spectrum of the bound-state energies when calculated in the units such that $\hbar^{2} /\left(2 \mu_{x}\right)=\hbar^{2} /\left(2 \mu_{y}\right)=1$.

\subsubsection{Dynamical regime of the centrally suppressed probability densities}

Due to the polynomial form of the potential we may also easily derive the harmonic-oscillator approximation of the potential near its other two local minima lying at $x= \pm \gamma$,

$$
V(x, y)=\left(\alpha^{2}-\beta^{2}\right) \gamma^{4}+12 \beta^{2} \gamma^{2} \xi^{2}+C^{2} \gamma^{2} y^{2}+\ldots, \quad x= \pm \gamma+\xi .
$$

Again, under the alternative assumption that these minima are global and pronounced (i.e., that $1 \ll \alpha \ll \beta$ ), we arrive at the alternative leading-order formula for the corresponding twicedegenerate (i.e., parity-degenerate) low-lying spectrum,

$$
E_{m, k}^{( \pm)}=\left(\alpha^{2}-\beta^{2}\right) \gamma^{4}+2 \sqrt{3}(2 m+1) \beta \gamma+C \gamma(2 k+1)+\ldots, \quad m, k=0,1, \ldots
$$

Naturally, the dominance of these energies in the low-lying spectrum of the whole system is given by the comparative smallness of $\alpha$ which still, by our assumptions, remains also very large in its absolute value.

Now we are prepared to compare the two alternative approximate formulate for the quantum ground state energies. Firstly, in the dominant $-\alpha$ regime we see that the ground-state energy (7) is $\beta$-independent, positive and only "slowly" growing as a quadratic function of $\alpha, E_{n}^{(0)} \approx$ $E_{n}^{(0)}(\alpha)=\mathcal{O}\left(\alpha^{2}\right)$. In contrast, in the dominant- $\beta$ regime the shape of the $\alpha$ - and $\beta$-dependent dominant-energy surface $E_{m, k}^{( \pm)}=E_{m, k}^{( \pm)}(\alpha, \beta)$ of Eq. (9) is, as a function of $\beta$ studied at a fixed $\alpha$, deeply negative, very steep $\left(E_{m, k}^{( \pm)}(\alpha, \beta)=\mathcal{O}\left(\beta^{6}\right)\right)$ and, in the vicinity of the end-of-the-domain limit $\beta \rightarrow \alpha^{+}$, even sign-changing (mainly due to the second, correction term $\mathcal{O}\left(\beta^{2}\right)$ which is positive).

Both of these parameter-dependence tendencies are monotonous. This is their important property which implies that the system in question will necessarily pass through its relocalization critical point at which the separate approximate subspectra (7) and (9) overlap. Due to the extremely steep, $\mathcal{O}\left(\beta^{6}\right)$ shape of the latter, $\beta$-dominant energy curve, the "critical" interval of 
$\beta \approx \alpha \gg 1$ (in which one would have to use brute-force numerical methods since both of our approximations (7) and (9) cease to be valid) remains extremely narrow. The change of the localization of the densities $|\psi(x, y)|$ becomes abrupt.

\subsubsection{Quantum relocalization phase transition}

In the light of our preceding considerations, the instant of the change of the type of dominance of densities $|\psi(x, y)|$ may be determined very easily. On our present level of precision it is sufficient to determine the instant of the intersection and coincidence of the two alternative candidates for the ground-state energy,

$$
E_{0}^{(0)}(\alpha) \approx E_{0,0}^{(+)}(\alpha, \beta)
$$

Up to the higher-order corrections the latter relation determines the relocalization catastrophe $(\mathrm{RC})$.

After a detailed inspection of our formulae we reveal that among the three coupling constants in the potential the two (viz., $A$ and $B$ ) were assumed, from the very beginning, large. For this reason, a decisive role determining the $\mathrm{RC}$ transition remains to be played by the third coupling $C$. In fact, this parameter measures the asymmetry of the potential so that its critical value $C=C^{(R C)}$ can also be treated as a function of the two other, freely variable dynamics-determining quantities $\alpha \gg 1$ and $\beta \gg 1$.

After an elementary algebra we obtain, from (10), our ultimate explicit formula

$$
C^{(R C)}=\left[(\alpha+\beta) \gamma^{3}-2 \sqrt{3}\right](\beta-\alpha) .
$$

Its last-step simplification

$$
C^{(R C)}=\left(\beta^{2}-\alpha^{2}\right) \gamma^{3}+\text { corrections }
$$

characterizes the dominant $\mathrm{RC}$ relationship resulting from the observation that in (11) the first term in the square brackets is dominant. Thus, we must have $\beta>\alpha \gg 1$, i.e., say, $\beta^{2}=$ $\alpha^{2}+q \alpha$ with a bounded auxiliary factor $q=\mathcal{O}(1)$. Nevertheless, an additional, very reasonable requirement of a parallel boundedness of $C^{(R C)}=\nu \alpha$ (with, say, $\nu=\mathcal{O}(1)$ ) leads to the amended estimate $q=\nu /\left(\sqrt{27} \alpha^{3}\right)$ so that in the critical regime the admissible difference between $\beta$ and $\alpha$ remains extremely small,

$$
\beta^{2}-\alpha^{2}=q \alpha=\mathcal{O}\left(\alpha^{-2}\right)
$$

This observation only reconfirms that, as expected, the process of the RC catastrophe is truly "abrupt". 


\section{Outlook}

In the quasi-classical limit of vanishing $\hbar \rightarrow 0$ (or, if you wish, of infinite mass $\mu \rightarrow \infty$ ), all of the low-lying quantum bound states described by Schrödinger Eqs. (1) or (2) become degenerate and localized strictly at the dominant minima of the potential. This may be perceived as a certain unidirectional correspondence between the genuine quantum equilibria and the Thom's classical (i.e., non-quantum) concepts of bifurcations alias catastrophes.

Formally speaking, the correspondence is not one-to-one because the classical theory admits also the potentials (called, in this context, Lyapunov functions) which are not asymptotically confining. In quantum world, on the contrary, one can easily construct various non-analytic potentials and systems exhibiting a catastrophic bifurcation behavior. Indeed, quantum Hamiltonians need not necessarily be defined in the Thom-inspired differential-operator form at all.

Our present letter demonstrated our belief that the parallels between the classical and quantum theory of catastrophes may be strengthened, anyhow. We followed the idea of searching for them under the reasonable assumption of using the most elementary polynomial forms of the local potential functions. In such an overall research project there emerged several key mathematical challenges. The main one may be seen in the fact that immediately after one moves from the onedimensional models of paper [1] to the much broader family of $D$-dimensional quantum systems with $D \geq 2$, the generic loss of the separability of the underlying Schrödinger equation seems to imply the necessity of a purely numerical localization and description of the catastrophes. In this sense our present results might be perceived as encouraging.

Let us also add that during our project we felt guided by the not quite expected mathematical friendliness of the one-dimensional constructions in Ref. [1]. Indeed, in that paper we managed to circumvent the two mutually interrelated difficulties of our catastrophe-simulation model-building, viz., the smeared, non-abrupt nature of the bifurcations (which is, in a way, typical for quantized systems), and an apparently purely numerical character of the solutions of Schrödinger equations, especially in the higher-degree polynomial-potential models.

In our present letter we came to a few equally encouraging observations. Our success has been achieved via our restriction of attention just to the very next, two-dimensional quantum systems, and to the dynamical regime characterized by the "sufficiently pronounced" local minima of $V(x, y)$ separated by the "sufficiently high" barriers. In this sense a similar success to be achieved, in the nearest future, at $D=3$ etc., need not be excluded.

For the sake of brevity we picked up just one of the most elementary nontrivial models, viz., the two-dimensional butterfly interaction (4). We were fortunate in revealing that even this next to elementary potential already exhibited many of the desirable phenomenological properties. Besides that, several challenging mathematical features of the model (like its non-separability) 
were found tractable. This proved promising and welcome, in particular, in the light of their quantum-theoretical relevance sampled, first of all, by the existence of the probability density relocalization phenomenon.

Marginally let us finally re-emphasize that in the phenomenologically most interesting regime of the competition for dominance between the multiple (i.e., in our model, three) pronounced local minima (approximated, with good precision, by the solvable harmonic-oscillator wells, and connected via the quantum tunneling of course) the determination of the instant of the "catastrophe" (i.e., of an abrupt change of the topology of the probability density $|\psi(x, y)|$ ) remained purely non-numerical.

\section{Acknowledgments}

The author acknowledges the support by the Faculty of Science of the University of Hradec Králové and, in particular, by the Excellence project 2212 PřF UHK 2020. 


\section{References}

[1] M. Znojil, Ann. Phys. 413, 168050 (2020).

[2] https://en.wikipedia.org/wiki/Catastrophe_theory

[3] R. Thom, Structural Stability and Morphogenesis: An Outline of a General Theory of Models. Addison-Wesley, Reading, 1989.

[4] J. Poston and I. Stewart, Catastrophe Theory and Its Applications, Pitnam, London, 1978;

[5] X. Krokidis, S. Noury and B. Silvi, J. Phys. Chem 101, 7277 (1997).

[6] V. I. Arnold, Catastrophe Theory. Springer-Verlag, Berlin, 1992. 